\title{
INVOLUTORIAL SPACE TRANSFORMATIONS ASSOCIATED WITH A RATIONAL RULED SURFACE*
}

\author{
BY L. A. DYE
}

1. Introduction. The present paper concerns various new types of Cremona involutorial transformations in $S_{3}$. Each one is a sample of an infinite category, and the concept can be extended to higher spaces.

Transformations obtained by joining corresponding points of curves or by associating the points of a curve with a projective pencil of surfaces have been studied from time to time and are sketched in the Encyklopädie, the Repertorium, and particularly in Selected Topics of Algebraic Geometry, including the Supplementary Report (Bulletin 63, National Research Council, 1928, No. 96, 1934). All these papers can be found in Topics, Chapters 8,9 , or Supplement, Chapters 4, 5 under the names Black, Carroll, Davis, DePaoli, Dye, Moffa, Montesano, Sharpe, and Snyder.

The types here discussed can not be put into any of the categories previously mentioned. The complex of lines defined by pairs $P P^{\prime}$ of associated points is not linear, is not special, and does not have any particular role in the problem. In most of the earlier cases it was formed by the secants to a given curve, or was linear. In the DePaoli types the lines $P P^{\prime}$ describe a congruence, each line containing an infinite number of pairs of conjugate points.

The procedure used in this paper is to establish a $(1,1)$ correspondence between the generators of a ruled surface $R$ and the surfaces of a pencil $|F|$. A general point $P$ of space selects a surface $F$ of the pencil and hence the associated generator $r$ of $R$. The plane $\pi$ determined by $r$ and $P$ is tangent to $R$ at a point $Q$. The line $P Q$ meets the surface $F$ in $P$ and a residual point $P^{\prime}$; any other intersections are accounted for by properly relating $|F|$ and $R$. The points $P, P^{\prime}$ are an associated pair in an involutorial transformation under which the pencil $|F|$ and the congruence of conics cut from $|F|$ by the planes $\pi$ are invariant.

\footnotetext{
* Presented to the Society, February 23, 1935, and November 30, 1935.
} 
2. Pencil of Quadrics through a $C_{4},(p=1)$. A pencil of quadric surfaces $\left|F_{2}\right|: C_{4}$ having an elliptic quartic curve in common, and a ruled surface $R_{n+1}: l^{n}$ of order $n+1$ with an $n$-fold line $l$ determine an involutorial transformation of order $8 n+5$. In this transformation there are two fundamental curves of the first species; one is the $C_{4}$ which is the base of $\left|F_{2}\right|$ and the other is a $C_{2 n+3}$ which is the locus of the piercing points of the lines $r$ and their associated quadrics. The image of each of these points is a conic and the conics generate the image surface $L_{8 n+4}$ of $C_{2 n+3}$.

There are $2 n-2$ torsal generators on $R_{n+1}$. The tangent planes along them cut conics from the associated quadrics which are parasitic, since every point of such a generator may be taken as a point $Q$ in the tangent plane, and hence the image of every point of the conic is the whole conic.

Through each generator $r$ there are two planes which cut degenerate conics from the associated quadric. If the point of contact $Q$ of such a plane is on one of the lines of the degenerate conic, then every point of the line will go into the whole line, and the line is parasitic. The number of such lines is $8 n+8$ and is determined by taking the complete intersection of two homoloidal surfaces.

In any plane $\pi$ the points of contact of the tangents drawn from $Q$ to the conic section lie on the invariant surface $K_{4 n+4}$ of the transformation. When $Q$ is a point of $C_{2 n+3}$, then that point will lie on both $K$ and $L$, and these surfaces will have contact along $C_{2 n+3}$.

Let the equation of $R_{n+1}$ be $a x_{2}+b x_{3}+c x_{4}=0$, where $a, b, c$ are binary forms of order $n$ in $x_{1}, x_{2}$. The equations of the line $l$ are $x_{1}=x_{2}=0$, and any plane through $l$ such as $\mu x_{1}-\lambda x_{2}=0$ cuts from $R_{n+1}$ a line $r$ whose equations may be written as follows: $\mu x_{1}-\lambda x_{2}=\bar{a} x_{2}+\bar{b} x_{3}+\bar{c} x_{4}=0$, where $\bar{a}=a(\lambda, \mu), \cdots$. The plane $\pi$ determined by a point $P(y)$ and the line $r$ is tangent to $R_{n+1}$ at the point $Q(z)$ which has the coordinates $\left(\lambda A_{0} R, \mu A_{0} R, \mu B_{0} R-n \bar{c} M\right.$, $\left.\mu C_{0} R+n \bar{b} M\right)$, where $A_{0} \equiv \bar{b}_{1} \bar{c}_{2}-\bar{b}_{2} \bar{c}_{1}, \cdots$, and $\bar{a}_{i} \equiv \partial a /\left.\partial x_{i}\right|_{\lambda, \mu}, \cdots$. The symbols $R, M$ represent the forms $\mu y_{1}-\lambda y_{2}, \bar{a} y_{2}+\bar{b} y_{3}+\bar{c} y_{4}$, respectively.

Let the equation of the pencil of quadrics be $\mu F_{2}^{\prime}-\lambda F_{2}^{\prime \prime}=0$. A point $P(y)$ determines a quadric $F_{2}$ which the line $P Q$ meets in the residual point $P^{\prime}\left(y^{\prime}\right)$, where $y_{i}^{\prime}=y_{i} L-z_{i} K$, and $L \equiv F_{2}(z)$, 
$K \equiv y_{i} \partial F(z) / \partial z_{i}$. The equations of the transformation are obtained by replacing $\lambda, \mu$ by $F_{2}{ }^{\prime}(y), F_{2}{ }^{\prime \prime}(y)$ in $y_{i}{ }^{\prime}$.

The table of characteristics of the transformation is

$$
\begin{aligned}
S_{1} \sim S_{8 n+5}: C_{4}{ }^{4 n+1}+C_{2 n+3}^{2+2 t} \\
C_{2 n+3} \sim L_{8 n+4}: C_{4}{ }^{4 n+1}+C_{2 n+3}^{2+t} \\
C_{4} \sim F_{16 n+8}: C_{4}{ }^{8 n+1}+C_{2 n+3}^{4+4 t}, \\
K_{4 n+4}: C_{4}{ }^{2 n+1}+C_{2 n+3}^{1+t} .
\end{aligned}
$$

The superscript $t$ is used to indicate that the multiplicity is due to contact; for example, the tangent planes to the two sheets of an $S_{8 n+5}$ through $C_{2 n+3}$ coincide at each point of $C_{2 n+3}$ with the tangent plane to $K_{4 n+4}$ at the point. The nature of the surface $F_{16 n+8}$ is obtained by finding the transform of a quadric $F_{2}$.

3. Pencil of Quadrics through the Line l. If the basis curve of the pencil of quadrics consists of the line $l$ and a residual space cubic, then the equation of $\left|F_{2}\right|$ may be written in the form $\left|F_{2}\right|=x_{1}(\alpha x)+x_{2}(\beta x)=0$, where $(\alpha x)$ is a linear homogeneous form in $x_{i}$ and $\alpha_{i} \equiv \mu \alpha_{i}^{\prime}-\lambda \alpha_{i}{ }^{\prime \prime}, \ldots$. The form $F_{2}(z)$ is composite and has the factors $A_{0} R[\lambda(\alpha z)+\mu(\beta z)] \equiv A_{0} R L$. The equations of the transformation are $y_{i}^{\prime}=y_{i} A_{0} R L-z_{i} K$, but $y_{1}^{\prime}=A_{0} R^{2}\left[(\beta z)-\lambda A_{0}\{\lambda(\alpha y)+\mu(\beta y)\}\right]$, and $y_{2}^{\prime}=A_{0} R^{2}[(\beta z)$ $\left.+\mu A_{0}\{\lambda(\alpha y)+\mu(\beta y)\}\right]$.

When a plane $\pi$ passes through $l$, the point of contact $Q(z)$ is at the intersection of $l$ and $r$. Such a plane cuts from $F_{2}$ a line meeting $l$ elsewhere than at $Q$. The image of the line is $Q$, and as $Q$ moves on $l$, the image lines generate the surface $R_{3}=0$ taken twice. At any point on $l$ the tangent planes to the $2 n$ sheets of a homoloidal surface coincide in pairs with the $n$ tangent planes to $M_{2 n+1}=0$ at that point, since the highest powers of $y_{3}, y_{4}$ have $\left(\bar{b} y_{3}+\bar{c} y_{4}\right)^{2}$ as a factor in the equation of an $S_{8 n+5}$, and $\left(\bar{b} y_{3}+\bar{c} y_{4}\right)$ in the case of $L_{4 n+5}$. The invariant surface $K_{4 n+4}$ does not have contact along $l$, but in the intersection of $K_{4 n+4}$, and on $S_{8 n+5}$ the line $l$ must be counted an additional $n$ times.

In this transformation each generator $r$ meets its associated quadric in a point on $l$, and one residual point. The locus of these residual points is a fundamental curve $C_{n+3},(p=0)$, which has the surface $L_{4 n+5}$ as its image. 
The $2 n-2$ pinch points of $R_{n+1}$ along $l$ are fundamental points of the transformation, since each of these points is associated with all the planes through its torsal generator. The image of a pinch point is the quadric surface associated with the torsal generator through the point. The equations of these quadrics are obtained by putting $A_{0}$ equal to zero.

The number of parasitic lines due to the point $Q(z)$ lying on a degenerate conic cut from $F_{2}$ by a plane $\pi$ is $4 n+5$, and the number of parasitic conics is $2 n-2$ as before. The table of characteristics of the transformation is

$$
\begin{aligned}
& S_{1} \sim S_{8 n+5}: l^{4 n+2}+n \bar{l}^{2}+C_{3}^{4 n+1}+C_{n+3}^{2+2 t}+(2 n-2) O_{i}^{4 n+5}, \\
& l \sim 2 R_{3}: l^{2}+C_{3}+C_{n+3}+(2 n-2) O_{i}^{2}, \\
& C_{n+3} \sim L_{4 n+5}: l^{2 n+2}+n \bar{l}+C_{3}^{2 n+2}+C_{n+3}^{1+t}+(2 n-2) O_{i}^{2 n+3} \text {, } \\
& C_{3} \sim F_{12 n+6}: l^{6 n+1}+n \bar{l}^{4}+C_{3}{ }^{6 n+2}+C_{n+3}^{2+2 t}+(2 n-2) O_{i}^{6 n+5}, \\
& (2 n-2) O_{i} \sim A_{4 n-4}: l^{2 n-2}+C_{3}{ }^{2 n-2}+(2 n-2) O_{i}^{2 n-1} \text {, } \\
& K_{4 n+4}: l^{2 n+2}+C_{3}^{2 n+1}+C_{3}^{1+t}+(2 n-2) O_{i}^{2 n+3},
\end{aligned}
$$

where the notation $\bar{l}$ is used to indicate contact along $l$.

4. Pencil of Cubic Surfaces with a Double Line. A pencil of cubic surfaces $\left|F_{3}\right|$ having a double line $l$ and a rational twisted quintic $C_{5}$ as a base is made projective with the pencil of planes $|p|$ through $l$. A general point $P$ will determine a surface $F_{3}$ and the plane $p$ which corresponds to $F_{3}$. The plane $p$ will cut from $F_{3}$ a line $r$ which generates a ruled quartic surface $R_{4}: l^{3}$. The pencil $\left|F_{3}\right|$ and the surface $R_{4}$ determine a transformation in the manner described in the introduction.

Let the equation of the pencil of cubic surfaces be $a x_{2}+b x_{3}$ $+c x_{4}=0$, where $a, b, c$ are binary quadratic forms in $x_{1}, x_{2}$, and $a_{i j} \equiv \mu a_{i j}^{\prime}-\lambda a_{i j}^{\prime \prime}, \cdots$. The equations of the planes $p$ and of the line $r$ have the same form as those used in $\$ 2$. The ruled quartic $R_{4}$ has the equation $x_{2} F_{3}^{\prime}-x_{1} F_{3}^{\prime \prime}=0$. The plane $\pi$ determined by a point $P(y)$ and the line $r$ is tangent to $R_{4}$ at the point $Q(z)$, where

$$
\left(z_{i}\right) \equiv(\lambda A R, \mu A R, \mu B R-2 \bar{c} M, \mu C R+2 \bar{b} M),
$$
in which $A \equiv A_{0}+2 A^{0}, \cdots$, and $A^{0} \equiv \bar{b}^{\prime} \bar{c}^{\prime \prime}-\bar{b}^{\prime \prime} \bar{c}^{\prime}, \cdots$.

The residual intersection of the line $P Q$ with the associated surface $F_{3}$ is the point $P^{\prime}\left(y^{\prime}\right)$, where 


$$
y_{i}^{\prime}=y_{i}\left(z_{i} \frac{\partial F(y)}{\partial y_{i}}\right) z_{i}\left(y_{i} \frac{\partial F(z)}{\partial z_{i}}\right) .
$$

Replacing $\lambda, \mu$ by $F^{\prime}(y), F^{\prime \prime}(y)$, we have $y_{i}^{\prime}=R^{3}\left(y_{i} A L-z_{i} K\right)$. The forms $L, K$ can be expressed as follows:

$$
\begin{aligned}
L \equiv & \bar{a}_{1} A^{0}+\bar{b}_{1} B^{0}+\bar{c}_{1} C^{0}-A^{0} N \\
N \equiv & 2 \mu y_{2} \alpha-\left(\mu y_{1}+\lambda y_{2}\right) \beta+2 \lambda y_{1} \gamma \\
K \equiv & N\left[2 \lambda y_{1} A_{22}-\left(\mu y_{1}+\lambda y_{2}\right) A_{12}+2 \mu y_{2} A_{11}\right] \\
& +2 \mu R D-A\left(\alpha y_{2}^{2}-\beta y_{1} y_{2}+\gamma y_{1}^{2}\right) .
\end{aligned}
$$

The letters $\alpha, \beta, \gamma$ represent the second-order determinants of the matrix

$$
\left\|\begin{array}{ccc}
a_{11}^{\prime} y_{2}+b_{11}^{\prime} y_{3}+c_{11}^{\prime} y_{4} & a_{12}^{\prime} y_{2}+b_{12}^{\prime} y_{3}+c_{12}^{\prime} y_{4} & a_{22}^{\prime} y_{2}+b_{22}^{\prime} y_{3}+c_{22}^{\prime} y_{4} \\
a_{11}^{\prime \prime} y_{2}+b_{11}^{\prime \prime} y_{3}+c_{11}^{\prime \prime} y_{4} & a_{12}^{\prime \prime} y_{2}+b_{12}^{\prime \prime} y_{3}+c_{12}^{\prime \prime} y_{4} & a_{22}^{\prime \prime} y_{2}+b_{22}^{\prime \prime} y_{3}+c_{22}^{\prime \prime} y_{4}
\end{array}\right\|,
$$

and $A_{i j}$ are the cofactors of $a_{i j}$ in $D \equiv\left|a_{11} b_{12} c_{22}\right|$.

When a point $Q$ is at the intersection of $r$ and $C_{5}$, the plane $\pi$ is tangent to both $R_{4}$ and $F_{3}$, since the line $r$ and the tangent to $C_{5}$ determine the plane $\pi$. The image of each point of $C_{5}$, when it plays this role, is the residual conic cut from $F_{3}$ by $\pi$. These conics generate the surface $L_{18}$. The $C_{5}$ has another image surface, however, since it also enters the transformation as a part of the base of $\left|F_{3}\right|$. This other image surface is obtained by transforming an $F_{3}$ and is of order 48 . Geometrically this surface is generated by the images of the four points of $C_{5}$ not on $r$ in any plane $\pi$.

In this transformation each line $r$ is a parasitic line and the surface $R_{4}$ generated by these lines factors out of the transformation three times. The line $l$ is a multiple parasitic line along which $S_{31}$ and $L_{18}$ have contact, since the highest powers of $y_{3}, y_{4}$ in their equations have $N_{6}$ as a factor.

The surfaces $L_{18}$ and $K_{16}$ have contact along $C_{5}$ as in the other transformations. The four pinch points $O_{i}$ of $R_{4}$ along $l$ are fundamental, and the tangent planes along the four torsal generators cut conics from the associated $F_{3}$ 's which are parasitic in the transformation. The number of parasitic lines is 14 as determined by the complete intersection of two $S_{31}$ 's. The table of characteristics of the transformation is 


$$
\begin{aligned}
S_{1} \sim & S_{31}: l^{19}+3 \bar{l}+C_{5}{ }^{10+2 t}+4 O_{i}{ }^{22} \\
C_{5} \sim & L_{18}: l^{11}+3 \bar{l}+C_{5}{ }^{6+t}+4 O_{i}{ }^{12} \\
& F_{48}: l^{28}+3 \bar{l}^{2}+C_{5}{ }^{15+5 t}+4 O_{i}{ }^{34} \\
4 O_{i} \sim & A_{12}: l^{8}+C_{5}{ }^{5}+4 O_{i}{ }^{9} \\
& K_{16}: l^{10}+C_{5}{ }^{5+t}+4 O_{i}{ }^{11} .
\end{aligned}
$$

5. Pencil of Cubic Surfaces with Two Double Points. The transformation to be developed here is similar to the one in the preceding section. A pencil of cubic surfaces $\left|F_{3}\right|$ through two double points $O_{i}$ contains the line through them and has a residual basis curve $C_{8}$ of order 8 with triple points at $O_{i}$. Each $F_{3}$ is tangent to a fixed plane along the line $l$ through $O_{i}$. These tangent planes cut lines $r$ from their associated $F_{3}$ and the lines $r$ generate a ruled quartic surface $R_{4}: l^{3}$.

Let $a x_{2}+b x_{3}+c x_{4}+\left(\mu x_{1}-\lambda x_{2}\right) x_{3} x_{4}=0$ be the equation of the $\left|F_{3}\right|$. The equations of the lines $r$ and the surface $R_{4}$ and the coordinates of the points $Q(z)$ are the same as those in the last section.

When $\lambda, \mu$ are replaced by $F_{3}^{\prime}(y), F_{3}^{\prime \prime}(y)$, the form $M$ is factorable into $R$ and another factor, which is of the sixth order in $y$ and contains a term $\left(y_{3} y_{4}\right)^{2}$. The factor $R^{3}$ comes off from the equations of the transformation and we have $y_{i}^{\prime}=y_{i} L-z_{i} K$.

The curve $C_{8}$ meets each generator $r$ in two points at which $R_{4}$ and the associated $F_{3}$ have contact. The image of $C_{8}$ consists of two surfaces; one is the $L_{30}$ due to the contact of $\left|F_{3}\right|$ with $R_{4}$ along $C_{8}$, and the other surface is an $F_{60}$ due to the $C_{8}$ being a part of the base of $\left|F_{3}\right|$.

The lines $r$ are all parasitic and the line $l$ is a multiple parasitic line. There are 24 parasitic lines which are not generators of $R_{4}$, and 4 parasitic conics. The surfaces $L_{30}$ and $S_{31}$ have contact along $l$, and the surfaces $K_{16}$ and $L_{30}$ have contact along $C_{8}$. The table of characteristics of the transformation is

$$
\begin{aligned}
S_{1} \sim & S_{31}: l^{6}+6 \bar{l}+C_{8}{ }^{10+2 t}, \\
C_{8} \sim & L_{30}: l^{6}+6 \bar{l}+C_{8}{ }^{10+t}{ }^{6} \\
& F_{60}: l^{11}+6 \bar{l}^{2}+C_{8}{ }^{19+5 t}, \\
& K_{16}: l^{4}+C_{8}{ }^{5+t} .
\end{aligned}
$$

The Citadel, Charleston, S. C. 